\title{
Measurement of three-dimensional stress field of RP model having a notch by using digital holography
}

\author{
Y. Tanaka, and S. Murata \\ Department of Mechanical and System Engineering, Kyoto Institute of Technology, Kyoto, Japan
}

\begin{abstract}
We measure three-dimensional stress field of RP model having a V-notch. RP model (Elastic modulues $\mathrm{E}=3317 \mathrm{MPa}, 9.3 \times 7.7 \times 50 \mathrm{~mm}^{3}$ having a V-notch) is made of acrylic transparent resin and tracer particle (averaged diameter: $60 \mu \mathrm{m}$ ) are dispersed. The model is subjected to the static load $(100 \mathrm{~N})$ at the middle. Firstly, three-dimensional deflection is measured by using digital holographic PTV (Particle Tracking Velocimetry). Finally, three-dimensional stress field of RP model is visualized as a differential value of the deflection field.
\end{abstract}

\section{Introduction}

For time-compression, Concurrent Engineering has evolved to integrate 3D modeling with CAD (Computer Aided Design), CAE (Computer Aided Engineering), CAM (Computer Aided Machining) and RP / PM (Rapid Prototyping / Manufacturing)[1]. CAE analysis is an integral part of timecompression technologies mainly based on Finite Element Analysis (FEA). However, the creation of accurate FEA models for complex engineering objects sometimes requires significant amounts of time and effort.

RP model are possible to fulfill the requirement and complement the CAE data [2]. RP model is directly produced from 3D CAD models by using NC machine or 3D printer. For example, the model made of transarent and birefringent material is used for a photoelastic analysis that it is useful to analysis stress or strain fields.

In three-dimensional photoelastic stress analysis for complex-shaped products, the model is initially stress frozen and then mechanically sliced for evaluating the stress field. The slices are analyzed by the mathematical analysis, and have provided three-dimensional stress distribution. Therefore, RP model is expendable. We should produce the RP model with respect to each experimental conditions. In practical use, this measurement technique is experimentally intensive.

We have proposed a measurement of three-dimensional stress field of RP model by using digital holography[3]. RP model is not sliced like three-dimensional photoelastic stress analysis. Consequently, this proposal method can observe a three-dimensional stress field repeatedly with respect to each experimental conditions.

In this paper, three-dimensional stress field of RP model having a V-notch is measured by using digital holography. The model is made of acrylic transparent resin and tracer particles are dispersed. Displacement of tracer particle dispersed in PR model is measured by digital holography[4]. Threedimensional concentration stress distribution are derived from this displacement. A large stress gradient near a V-notch is visualized.

a e-mail: tyohsuke@kit.ac.jp

This is an Open Access article distributed under the terms of the Creative Commons Attribution-Noncommercial License 3.0, which permits unrestricted use, distribution, and reproduction in any noncommercial medium, provided the original work is properly cited. 


\section{Record and reconstruction of digital in-line holography}

Tracer particles dispersed in RP model are measured by digital holography. Digital holography is known as a three-dimensional measurement. Hologram pattern obtained by digital holography is experimentally observed by CCD camera and numerically reconstructed by a personal computer. Small particle such as tracer particle is particularly measured by using in-line holography [4]. A feature of in-line holography is the alignment which installs observed particles and optical system in the same straight line as shown in Fig. 1.

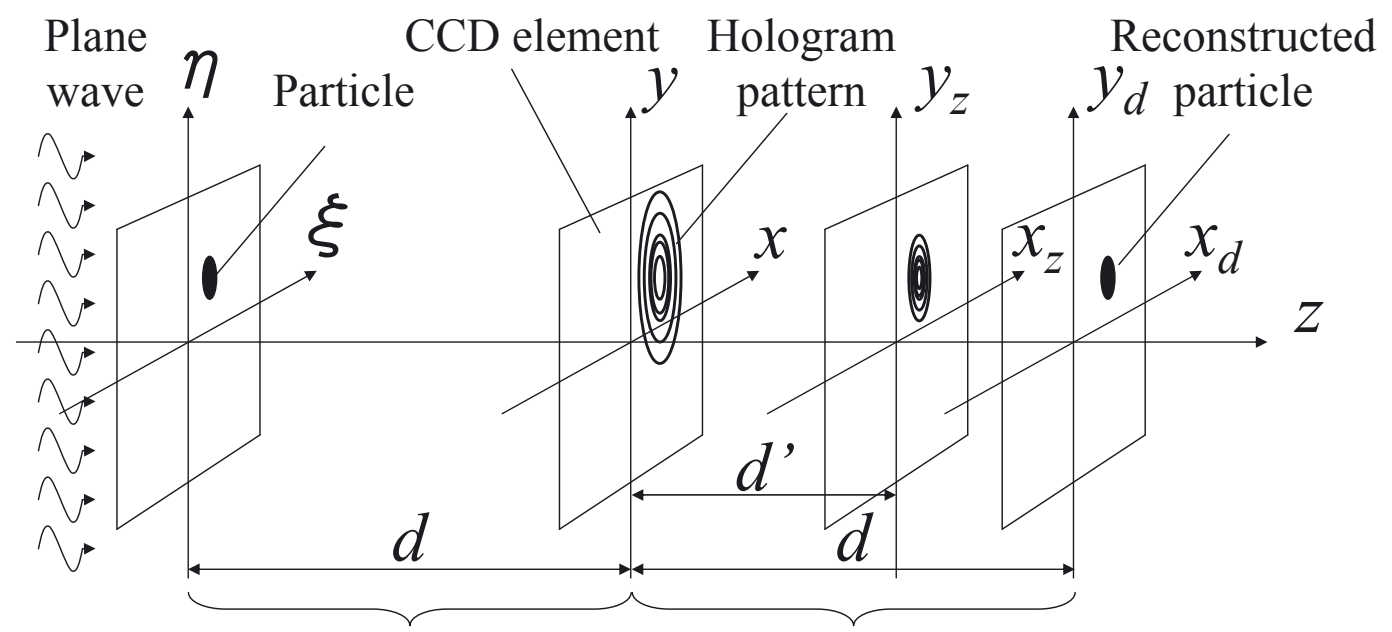

Experimental recording Numerical reconstruction

Fig. 1. Positional relation between recording and reconstruction of hologram pattern.

In the process of recording hologram pattern of tracer particle, the particle which located on $\xi-\eta$ plane is illuminated by plane wave (reference wave) from left-hand-side. Reference wave and Object wave diffracted on the surface of the particle are recorded on the CCD element as light intensity $I_{d}(x, y, 0)$ of hologram pattern. CCD element is located on $x-y$ plane at the distance $d$ from the particle.

In the process of reconstruction, the recorded hologram pattern is numerically reconstructed as digital data by a personal computer. Light amplitude distribution $h_{z}\left(x_{z}, y_{z}\right)$ located at an arbitrary position $z=d^{\prime}$ is expressed as:

$$
\begin{aligned}
h_{z}\left(x_{z}, y_{z}\right) & =\frac{1}{j \lambda} \int_{-\infty}^{\infty} \int_{-\infty}^{\infty} I_{d}(x, y, 0) \frac{\exp \left(j \frac{2 \pi L}{\lambda}\right)}{L} d x d y \\
L & =\sqrt{d^{\prime 2}+\left(x_{z}-x\right)^{2}+\left(y_{z}-y\right)^{2}}
\end{aligned}
$$

where, $j$ and $\lambda$ are imaginary unit and wavelength of plane wave, respectively. $L$ are the distance of two points on each plane between $x-y$ plane and $x_{z}-y_{z}$ plane. Light intensity $I_{z}\left(x_{z}, y_{z}\right)$ is derived from Eq. (1) as follows:

$$
I_{z}=h_{z} h_{z}^{*}
$$

From above equations, the tracer particle is reconstructed at $z=d$ as light intensity $I_{d}\left(x_{d}, y_{d}\right)$. The light intensity is locally a minimum at this position. Therefore, this position is detected as a threedimensional position of tracer particle. 


\section{Experimental method for three-dimensional stress field of RP model}

\subsection{Optical setup for recording of hologram pattern}

Figure 2 shows the optical setup for recording hologram pattern. All optical equipment and RP model are set up in line on an optical rail.

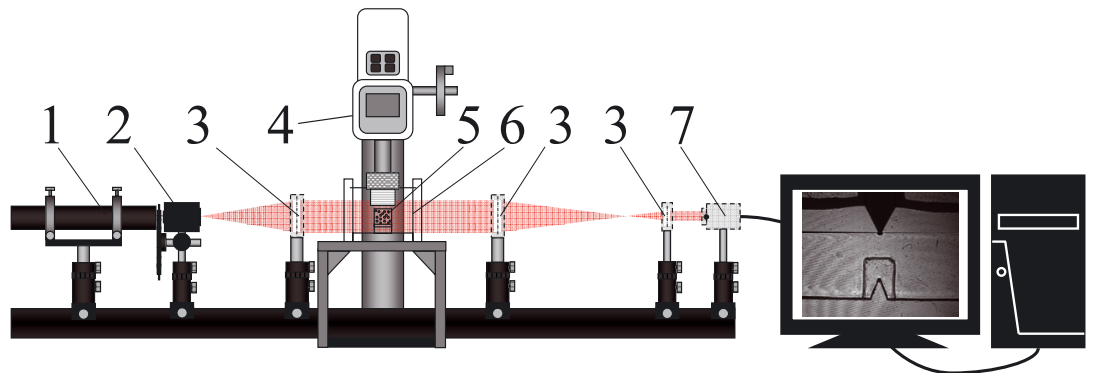

1. He-Ne Laser 3. Collimated lens 5. RP model 7. CCD without lens

2. Spatial fileter 4. Push pull gauge 6. Water tank

Fig. 2. Optical setup for recording of hologram pattern.

Tracer particles in RP model are illuminated by plane wave (diameter: $50 \mathrm{~mm}$ at the first collimated lens) which generated with He-Ne laser (Wavelength: $632.8 \mathrm{~nm}$ ). Spatial noise of the wave is eliminated by a spatial filter. The diameter of the wave is reduced to $13.7 \mathrm{~mm}$ with final collimated lens. The light intensity of hologram pattern is recorded by the CCD camera (Resolution: $2048 \times 2048$ pixel $^{2}$, Cell size: $3.45 \times 3.45 \mu \mathrm{m}^{2}$, XC-8500, Sony).

In order to suppress the different refractive index between the model and surrounding air, the model is installed in the acrylic water tank which is filled with a refractive-index matching liquid with the same value of the model' s refractive index. Aqueous solution of Sodium iodide is used as a matching liquid.

\subsection{Loading test of RP model having a V-notch}

RP model having a V-notch is made of acrylic transparent resin (Refractive index: 1.4883, Elastic modulus E $=3317 \mathrm{MPa}$, Acrybond, Mitsubishi Rayon) and tracer particles (Averaged diameter: 60 $\mu \mathrm{m}$, SP20SS, Mitsubishi Chemical) are uniformly dispersed as shown in Fig. 3.
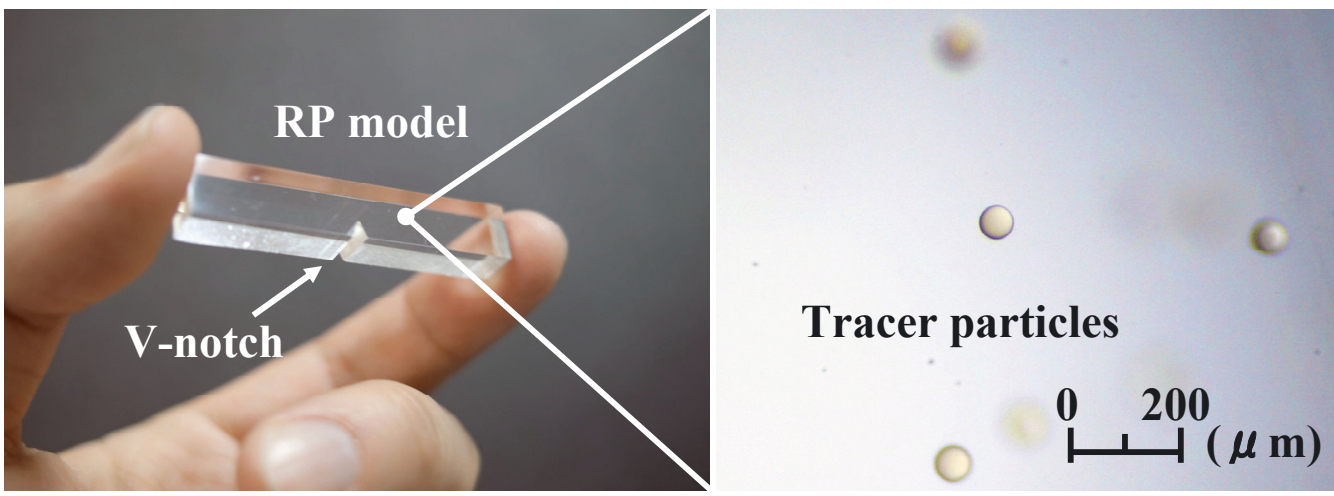

Fig. 3. RP model having a V-notch and dispersed tracer particles. 
We execute loading test of RP model having a V-notch as sketched in Fig. 4 . The model $(9.3 \times 7.7 \times 50$ $\left.\mathrm{mm}^{3}, l=15 \mathrm{~mm}, n=2 \mathrm{~mm}\right)$ is placed on two supports and subjected to a concentrated force $(\mathrm{P}=100$ $\mathrm{N})$ at the middle. The coordinate origin is located at the center of gravity. The observation volume is $25.7 \times 25.7 \times 15.8 \mathrm{~mm}^{3}$. The deflection of the model is obtained by each dispersed particle's positions between unloaded and loaded model.

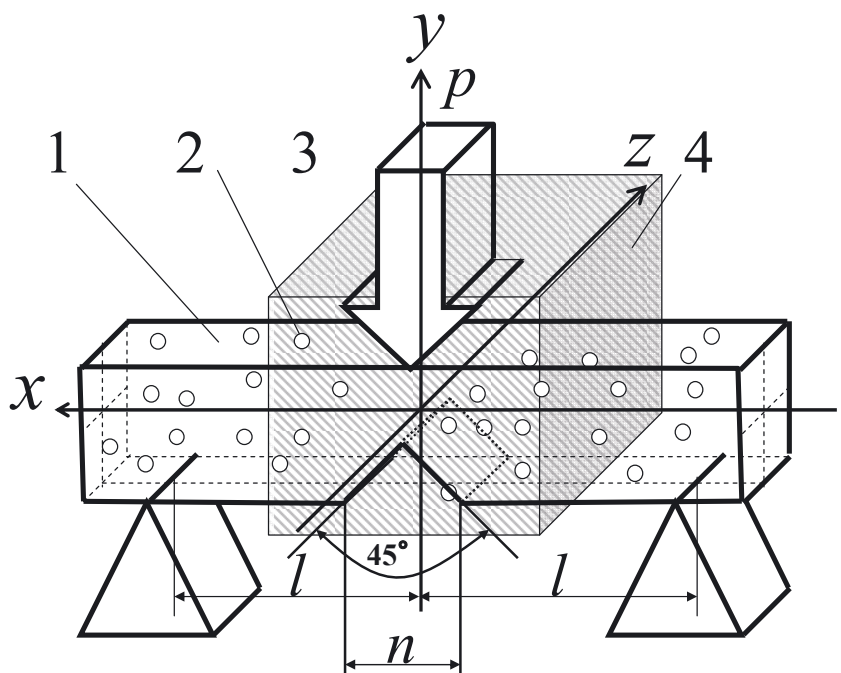

1. RP model with tracer particles

2. Tracer particle
3. Index matching liquid

4. Observation volume

Fig. 4. Loading test of RP model having a V-notch.

\subsection{Derivation Procedure for 3D stress field}

Figure 5 shows derivation procedure for 3D deflection field. This procedure is based on 3D PTV (Particle Tracking Velocimetry) techniques used for fluid measurement [5]. Firstly, three-dimensional vector field of tracer particles dispersed in RP model is measured. Finally, the stress field is derived as differential value of the vector field. This procedure is divided into six steps.

In Step 1, a pair of hologram patterns of unloaded and loaded model are recorded by using CCD camera. The hologram pattern is reconstructed, and the position of each particle are detected as a minimum intensity in 3D reconstructed volume [4] in Step 2. In Step 3, the vector field is calculated from pair of particle positions by using SOM (Self Organizing Map) [6].

Erroneous vectors are removed from the vector field [7] in Step 4. The vectors are rearranged to nearest grid and are applied LER (Laplace Equation Rearrangement) [8] in Step 5.

In step 6, Substituting displacement vector $\mathbf{U}$ which obtained in Step 5 into Eq. (4) yields strain field $\varepsilon$. Stress field $\sigma$ is derived from Eq. (5).

$$
\begin{gathered}
\varepsilon\left(\varepsilon_{x}, \varepsilon_{y}, \varepsilon_{z}\right)=\partial \mathbf{U} / \partial \mathbf{x}=(\partial u / \partial x, \partial v / \partial y, \partial w / \partial z) . \\
\sigma\left(\sigma_{x}, \sigma_{y}, \sigma_{z}\right)=E \cdot \varepsilon=\left(E \cdot \varepsilon_{x}, E \cdot \varepsilon_{y}, E \cdot \varepsilon_{z}\right) .
\end{gathered}
$$


Step 1 Recording of Hologram
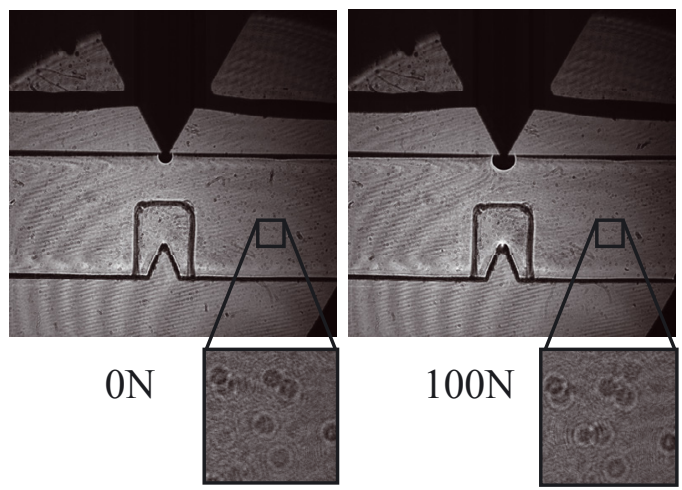

Step 4

Removal of erroneous vectors

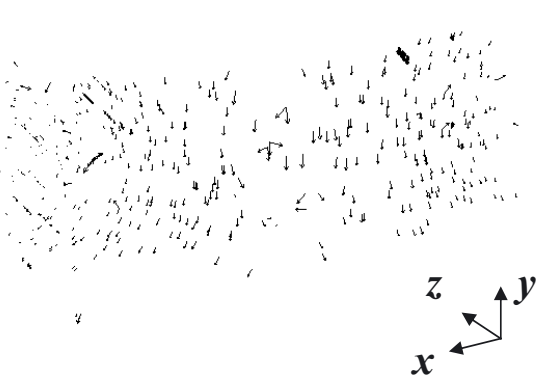

Step 2 Reconstruction of Hologram
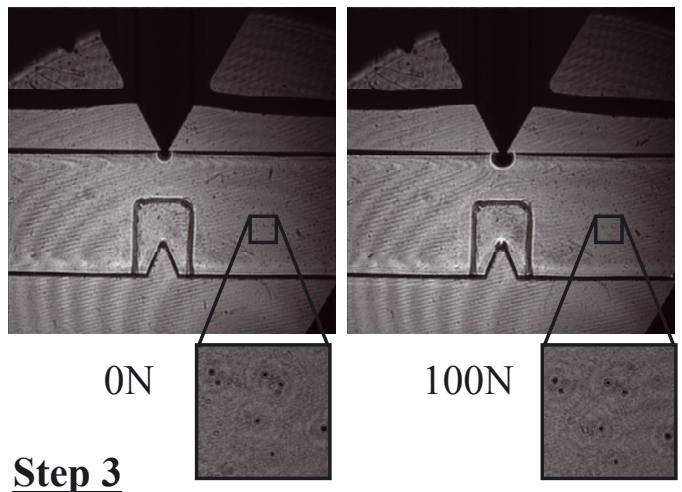

Step 5

\section{LER}

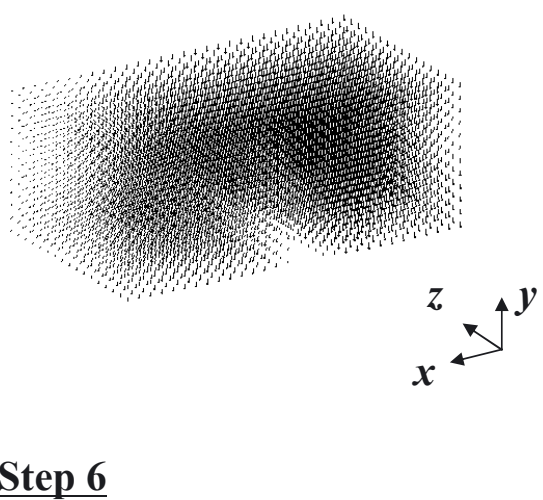

SOM

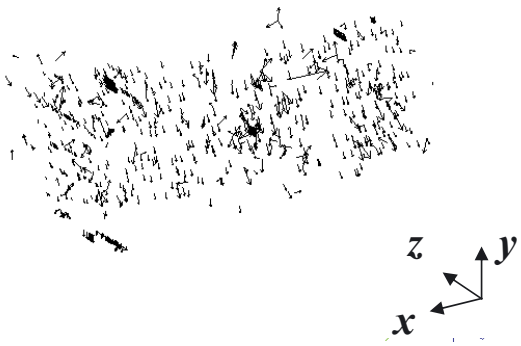

Fig. 5. Derivation procedure for three-dimensional stress field. 

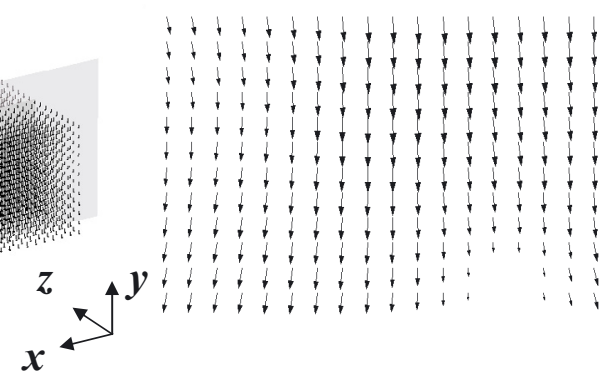

\section{(a) Three-dimensional deflection field of $u, v(z=0)$}
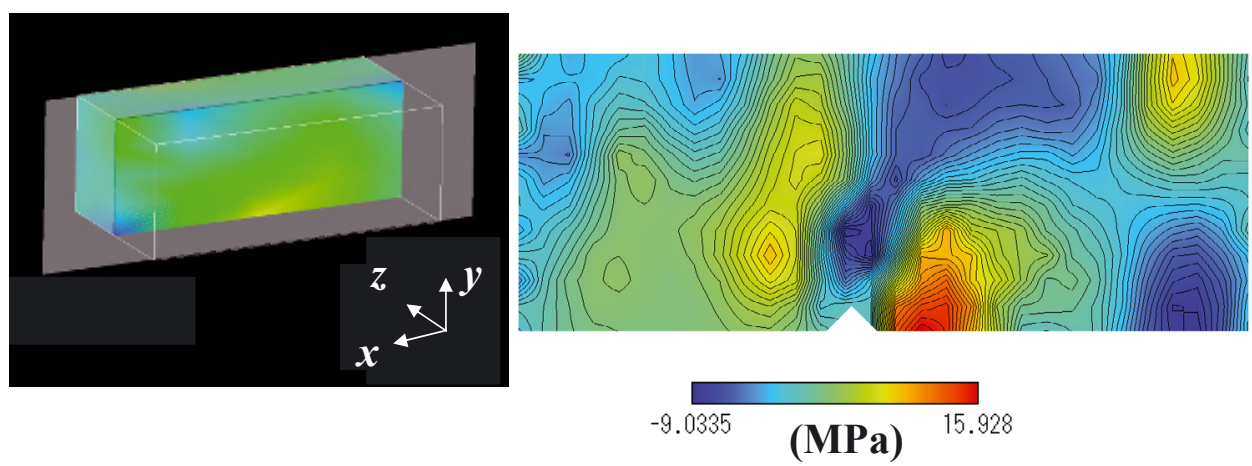

(b) Distribution of $\sigma_{x}(z=0)$
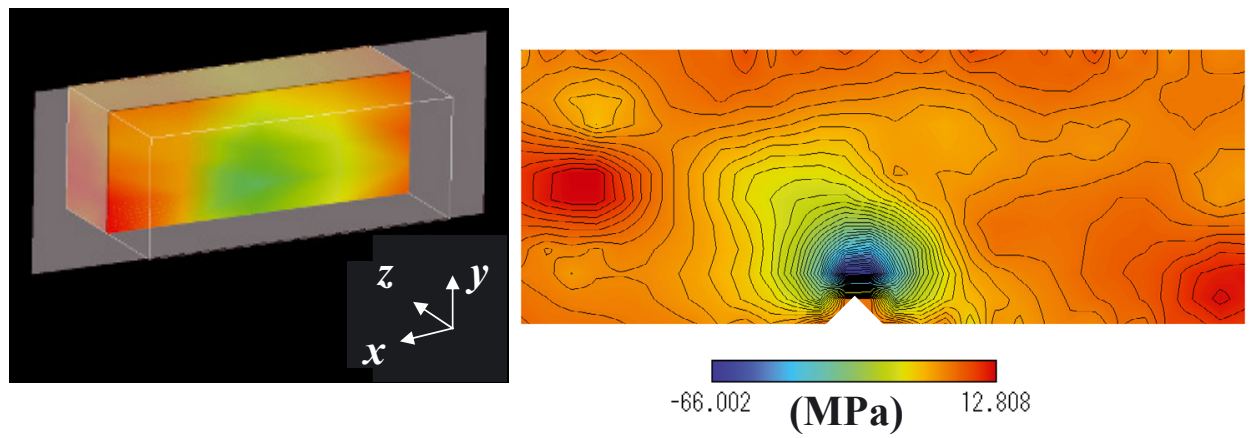

(c) Distribution of $\sigma_{y}(z=0)$

Fig. 6. Three-dimensional deflection and stress fields. 


\section{Three-dimensional stress field}

Three-dimensional deflection field $u, v$ and stress field $\sigma_{x}, \sigma_{y}$ are presented in Fig. 6. Here, we consider observation volume as grid points $30 \times 11 \times 17$ in $x, y, z$ directions respectively. Central differences are used at each grid points. In (a) of Fig. 6, vector field in RP model is measured by Holographic PTV as an experimental fluid mechanics. It is found that vectors near a V-notch are resolved. A large stress gradient near a V-notch is also visualized as shown in (b) and (c) of Fig. 6. However, the proposed method indirectly measures three-dimensional stress field. The model can be not only measured repeatedly under several conditions but also can be measured with dynamic load.

\section{Conclusion}

We demonstrate the measurement of three-dimensional stress field of RP model having a notch by using digital holography. The main results are summerized as follows:

1. Deflection field of RP model is visualized by using digital holography.

2. Stress concentration of RP model is visualized by using digital holography.

\section{Acknowledgment}

This work was supported by a Grant-in-Aid for Young Scientists (Start up) (No. 2086005) from Japan Society for the Promotion of Science.

\section{References}

1. D.T. Pham, and S.S. Dimov, Rapid Manufacturing (Springer, 2001) 6-8.

2. K. Ramesh, Digital Photoelasticity (Springer, 2000) 348-367.

3. Y. Tanaka and M. Murata, Proc. 4th ISEM '09-Niigata (2009) 85.

4. S. Murata and N. Yasuda, Optics \& laser Technology (2000) 567-574.

5. M. Raffel, C. Willert, S. Wereley and J. Kompenhans, Particle Image Velocimetry, 2nd edition, Springer, (2007) 209-239.

6. K. Ohmi, Proc., PSFVIP-4 (2003) F4006.

7. M. Raffel, C. Willert, S. Wereley and J. Kompenhans, Particle Image Velocimetry, 2nd edition, Springer, (2007) 178-188.

8. Y. Murai, T. Ido, M. Ishikawa and F. Yamamoto, Trans. JSME (B) (1997) Vol. 64 109-116 (in Japanese). 